\title{
The Effect of Cold Atmospheric Plasma Treatment on Cancer Stem Cells
}

\author{
Barry Trink, ${ }^{1}$ Michael Keidar, ${ }^{2}$ Jerome Canady, ${ }^{1}$ Yeela Shamai, ${ }^{3}$ \& \\ Maty Tzukerman ${ }^{3, *}$ \\ 1Jerome Canady Institute for Advance Biological and Technical Science, Plasma Medicine Life \\ Science, Takoma Park, MD, USA; ${ }^{2}$ Departments of Mechanical and Aerospace Engineering, \\ The George Washington University, Washington, DC, USA; ${ }^{3}$ Rappaport Faculty of Medicine and \\ Research Institute, Technion-Israel Institute of Technology, Rambam Medical Center, Haifa, Israel \\ *Address all correspondence to: Maty Tzukerman, Molecular Medicine Laboratory, B. Rappaport Faculty of Medicine, \\ Technion-Israel Institute of Technology, P.O.B. 9649, Bat Galim, Haifa 31096, Israel; Tel: +972-4-8295277/81; Fax: \\ +972-4-8512380; E-mail: bimaty@techunix.technion.ac.il; Michael Keidar keidar@gwu.edu), Departments of Mechani- \\ cal and Aerospace Engineering, The George Washington University. Science \& Engineering Hall, 800 22nd St, NW, \\ Washington, DC 20052, USA.; Tel: 202-994-6929; Fax: 202-994-0238
}

\begin{abstract}
Intratumoral heterogeneity challenges existing paradigms for anticancer therapy. Accumulating evidence demonstrates that the model of cancer stem cells (CSCs) and the model of clonal evolution mutually contribute to intratumoral heterogeneity, as CSCs themselves undergo clonal evolution. The limitation of conventional anticancer therapies may lead to treatment failure and cancer recurrence, mainly due to drug resistance and self-renewal capacities of CSC. These two factors are responsible for resistance to standard oncology treatments. In this study, we examine cold atmospheric plasma (CAP) treatment of CSC in vitro. We demonstrate that two types of heterogeneous CSC populations derived from a single patient tumor are sensitive to the effects of plasma treatment. Surprisingly, the more aggressive CSC population (C13) was more sensitive to CAP treatment than the less aggressive type (C12).
\end{abstract}

KEY WORDS: ovarian cancer, ovarian cancer stem cells, heterogeneity of cancer stem cells, CAP treatment in cancer

\section{INTRODUCTION}

Plasma is an ionized gas that is generated in high-temperature laboratory conditions. Recent developments in plasma physics research has led to the production of cold plasmas with ion temperature close to room temperature. ${ }^{1-3}$ Initial studies demonstrated the nonaggressive nature of the cold plasma, whereby plasma can interact with organic materials without causing thermal/electrical damage to the cell surface. These developments opened up new avenues for plasma applications in biological settings including wound healing, disinfection, and more recently in cancer research., ${ }^{4,5}$ This has led to the development of a new field in biological research known as plasma medicine.

Plasma medicine is a relatively new scientific field which emerged from research in the application of low-temperature (or cold) atmospheric plasmas in bioengineering. ${ }^{1-4}$ Cold atmospheric plasma (CAP) interacts with tissue in ways that allow targeted cell removal without necrosis, which is cell disruption. CAP affects cells via a programmable process called apoptosis, ${ }^{1-4}$ a multistep process leading to cell death. Recent cold plasma 
therapy studies, both in vivo and in vitro, demonstrated that apoptosis occurs in bacterial and mammalian cells including various types of cancer cells. The first in vivo demonstration of CAP anticancer potential was performed by Vandamme et al. ${ }^{6,7}$ on human U87 glioblastoma xenotransplants. This study indicated that treatment over multiple days was effective in reducing tumor volume and increasing survival time through apoptosis mediated by ROS-reactive oxygen species. In another study the antitumor action of CAP was demonstrated on syngeneic mouse melanoma and heterotopic human bladder cancer xenograft models. ${ }^{8}$ The ability of CAP to ablate the tumor in a single treatment was one of the most interesting results demonstrated. In particular, tumors about $5 \mathrm{~mm}$ in diameter were ablated after approximately 2 minutes in a single treatment.

Nevertheless, it is now widely appreciated that a single tumor is basically comprised of heterogeneous cell populations, each of which display a diverse cellular morphology, phenotypic expression, tumor initiation capacities, and inherent or acquired resistance to anticancer drugs. ${ }^{9-12}$

The aggressiveness and ingenuity of human cancers emanate mainly from such complex intratumoral heterogeneities, which in turn have been attributed to genetic and epigenetic changes coupled with adaptive responses to the tumor microenvironment. Accumulating evidence demonstrates that the cancer stem cell (CSC) model and the clonal evolution model mutually contribute to intratumoral heterogeneity, as CSCs themselves undergo clonal evolution. ${ }^{13-16}$ The continuous accumulation of mutations generates heterogeneity of cells within a solid tumor and its metastases, and they may reflect the process whereby certain subsets of tumor cells become more aggressive during tumor progression.

The limitation of conventional anticancer therapies may lead to treatment failure and cancer recurrence mainly due to drug resistance and self-renewal capacities of CSCs, which are responsible for resistance to standard oncology treatments. ${ }^{17}$ Therefore, to overcome the ineffectiveness of traditional cancer therapies that lead to tumor recurrence and metastasis, it is important to develop efficient anticancer treatments. The combination of conventional anticancer drugs with treatments targeting CSCs may offer a promising strategy for management and eradication of different types of cancers. ${ }^{18}$

CAP was previously shown to be a potential novel therapy for the treatment of cancer ${ }^{6-8,19}$ In addition, previous research demonstrated that cancer cells are more sensitive than normal cells to the effects of plasma. ${ }^{20}$ This offers a promising alternative to conventional therapies that have harmful side effects. It was recently demonstrated that cancer initiating cells (CICs) underwent apoptosis at a comparable level to non-CICs.21 For this reason, we sought to examine the effect of CAP on two different ovarian cancer stem cells derived from a single tumor to determine its feasibility in eradication of CSC in vitro. 


\section{MATERIALS AND METHODS}

\section{A. Cold plasma treatments}

Cold plasma treatments were carried out at high-voltage (HV) in the range of $3-5 \mathrm{kV}$, a frequency of $20 \mathrm{kHz}$, helium flow in the range 10-20 L-min-1, distance from plasma source to cells of about $1 \mathrm{~cm}$, and treatment durations of about 30 seconds.

Electrical measurements were performed with a Tektronix TDS3014C Digital Phosphor Oscilloscope. Emission spectra were recorded with an optical fiber, which was connected to a fiber optic spectrometer (EPP2000-HR, Stella Net, measurements can be made in UV-VIS-NIR ranges from 190-2200 nm). The feeding gas was helium.

\section{Derivation of ovarian cancer cell subpopulations}

After written informed consent was obtained, ascites fluid was collected from a 64-yearold patient diagnosed with stage IV ovarian clear cell carcinoma. The protocol was approved by the institutional Ethics Review Committee of the Rambam Medical Center. Six different cancer cell subpopulations, clonally expanded from a single cell, including cancer cell subpopulations (CCSPs) $\mathrm{C} 12$ and C13, were derived from the ovarian ascites containing malignant cells and propagated in culture as previously described. Although maintained in culture for more than 6 years, cell cultures are repeatedly initiated from frozen stocks every 3-4 months, and the CCSPs durably and consistently maintain the bona fide ovarian cancer characteristics, CSC characteristics, and xenografted tumor histological phenotype. ${ }^{9,22}$

\section{CAP sensitivity assay}

CCSP C12 and C13 cancer stem cells were plated in triplicate on fibronectin-coated 12 -well plates ( $5^{\prime} 103$ cells per well) in RPMI-1640 medium supplemented with $20 \%$ fetal bovine serum (FBS), 1\% penicillin/streptomycin, and 1\% glutamine. Medium was replaced every other day. Cells were treated with CAP for 1, 2, and 3 minutes at days 5,6 , and 8 following seeding. For control, untreated cells and helium-treated cells were used. All cells were harvested on day 11 (from seeding) and counted.

\section{RESULTS}

\section{A. Cold plasma source}

The cold plasma source developed at George Washington University ${ }^{23}$ is equipped with a pair of HV electrodes, a central electrode, and an outer ring electrode as shown in Figure 1. Electrodes are connected to a secondary coil of HV resonant transformer operating at a voltage of $2-5 \mathrm{kV}$ and a frequency of about $30 \mathrm{kHz}$, with a helium flow rate of 5-10 L- $\mathrm{min}^{-1}$. The length of the visible plasma jet was approximately $5 \mathrm{~cm}$ and was

Volume 5, Issue 1, 2015 


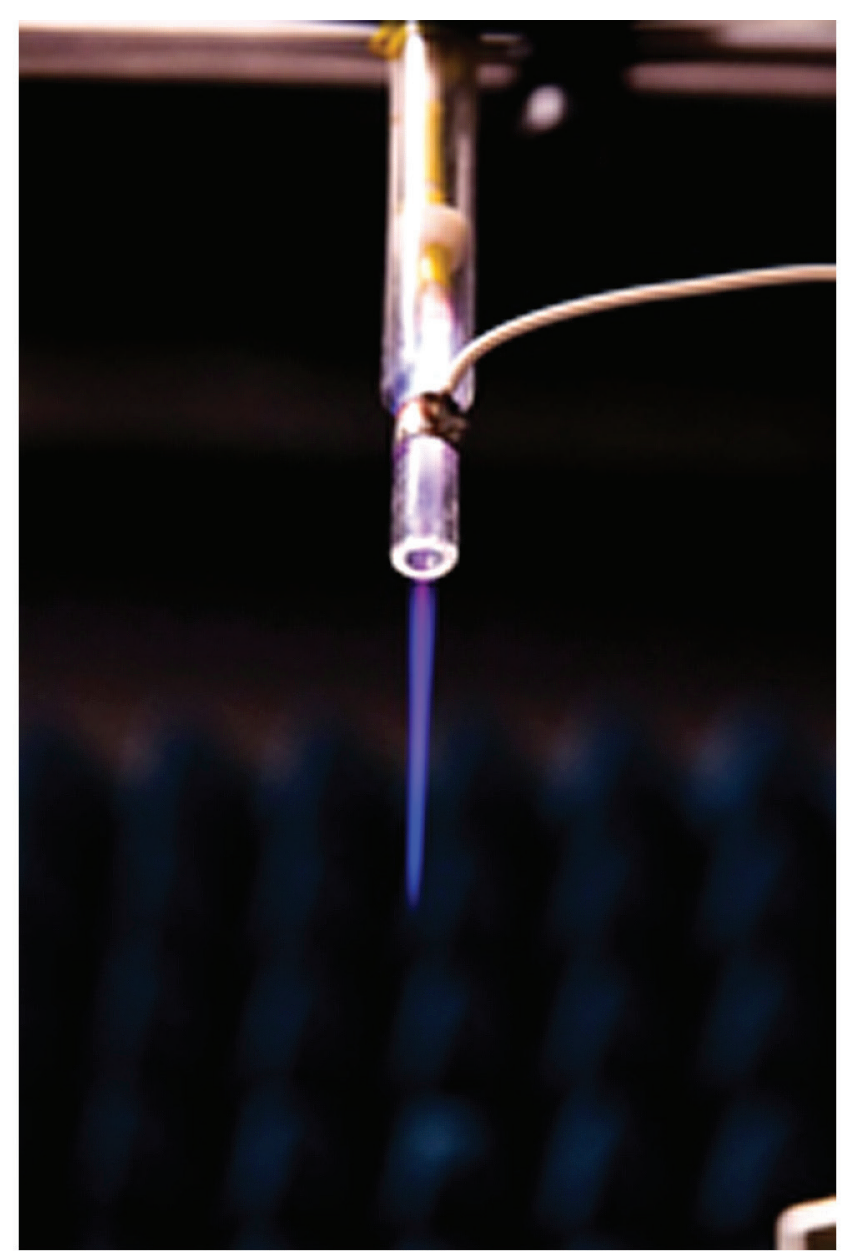

FIG. 1: Cold atmospsheric plasma (CAP) jet.

well collimated along the entire length. According to previous studies ${ }^{23}$ the plasma jet is discontinuous and represents a series of propagating plasma bullets.

\section{B. Ovarian cancer-derived heterogeneous cancer stem cells}

Ovarian clear cell carcinoma (OCCC) is characterized by striking intratumoral morphologic heterogeneity, including some cells with features of advanced ovarian structural variation and other cells with features of tumorigenic differentiation (e.g., invasion, proliferation) and corresponding cell surface and intracellular marker heterogeneity. ${ }^{24-28} \mathrm{We}$ have isolated and characterized six different CCSPs from a tumor of a single patient, and demonstrated niche-dependent tumorigenic capacities and histological phenotypes that cumulatively recapitulate the full spectrum of tumor heterogeneity. ${ }^{22}$ The six CCSPs, 
each clonally expanded from a single cell, demonstrate striking intratumoral phenotypic heterogeneity that is dynamically dependent on the tumor growth microenvironment. The six CCSPs were characterized as ovarian CSC according to their functional and phenotypic expression of CD44+CD24+EpCAM+ and ALDH1 activity (Fig. 2). ${ }^{9,22}$

To examine the effect of CAP on CSCs we focused on two distinct CCSPs, C12 and $\mathrm{C} 13$, which exhibit the extremes of tumorigenic phenotypic attributes and nichedependent self-renewal capacity., ${ }^{9,22} \mathrm{C} 12$-derived tumors are characterized by an abundance of highly differentiated ovarian structures, whereas C13-derived tumors exhibit poor ovarian structural differentiation..$^{22}$ In addition, C13 preserves its capacity for self-renewal as demonstrated by in vivo perpetuation of tumorigenic cancer cells, both in the murine and in the hESC-based in vivo model. However, $\mathrm{C} 12$ fails to perpetuate tumorigenic cells in the murine tissue, but it generates highly aggressive and invasive tumors within the human embryonic stem cells (hESC)-based in vivo model. ${ }^{9}$ In the light of this striking effect, we will examine the effect of CAP in eradication of these two populations of patient-derived ovarian tumor CSCs.

\section{In vitro cold plasma treatment of cancer stem cells}

To examine the effect of plasma treatment on CCSPs C12 and C13 in vitro, cells were seeded on day 1 as described in Table 1 . The cells were treated with plasma on days 5 , 6 , and 8 for 1,2, or 3 minutes and counted on day 11 after seeding. Controls were either untreated cells or cells treated with helium for 3 minutes. As seen in Table 1, the results demonstrate that both CCSPs C12 and C13 were sensitive to plasma to varying degrees. The $\mathrm{C} 13$ cells were very sensitive to the plasma treatment, whereas the $\mathrm{C} 12$ cells were less sensitive (Fig. 3). Taken together, these results suggest that CAP treatment could effectively eradicate CSC in patients with ovarian tumors.

TABLE 1: Ovarian cancer stem cells treated with cold atmospheric plasma in vitro.

\begin{tabular}{l|c|c|c|c|c|c|c|c}
\hline & \multicolumn{2}{|c|}{ Cell Number } & \multicolumn{2}{c|}{ Cell Number (average) } & \multicolumn{2}{c|}{ STDEV } & \multicolumn{2}{c}{ \% Survival } \\
\hline & $\mathbf{c 1 2}$ & $\mathbf{c 1 3}$ & $\mathbf{c 1 2}$ & $\mathbf{c 1 3}$ & $\mathbf{c 1 2}$ & $\mathbf{c 1 3}$ & $\mathbf{c 1 2}$ & $\mathbf{c 1 3}$ \\
\hline Control & 141,000 & 85,333 & 143,500 & $87,999.5$ & 3535.534 & 3771 & 100.00 & 100.00 \\
\hline & 146,000 & 90,666 & & & & & & \\
\hline Helium & 114,000 & 37,000 & $115,000.00$ & 35,833 & 1414.214 & 1650.387 & 80.14 & 40.72 \\
\hline & 116,000 & 34,666 & & & & & & \\
\hline 1 min & 103,333 & 13,500 & $105,833.00$ & $12,866.5$ & 3535.534 & 895.9043 & 73.75 & 14.62 \\
\hline & 108,333 & 12,233 & & & & & & \\
\hline 2 min & 25,000 & 5200 & $24,312.5$ & 4800 & 972.2718 & 565.6854 & 16.94 & 5.45 \\
\hline & 23,625 & 4400 & & & & & & \\
\hline 3 min & 14,933 & 1000 & $13,299.50$ & 943 & 2310.118 & 80.61017 & 9.27 & 1.07 \\
\hline & 11,666 & 886 & & & & & & \\
\hline
\end{tabular}

Volume 5, Issue 1, 2015 


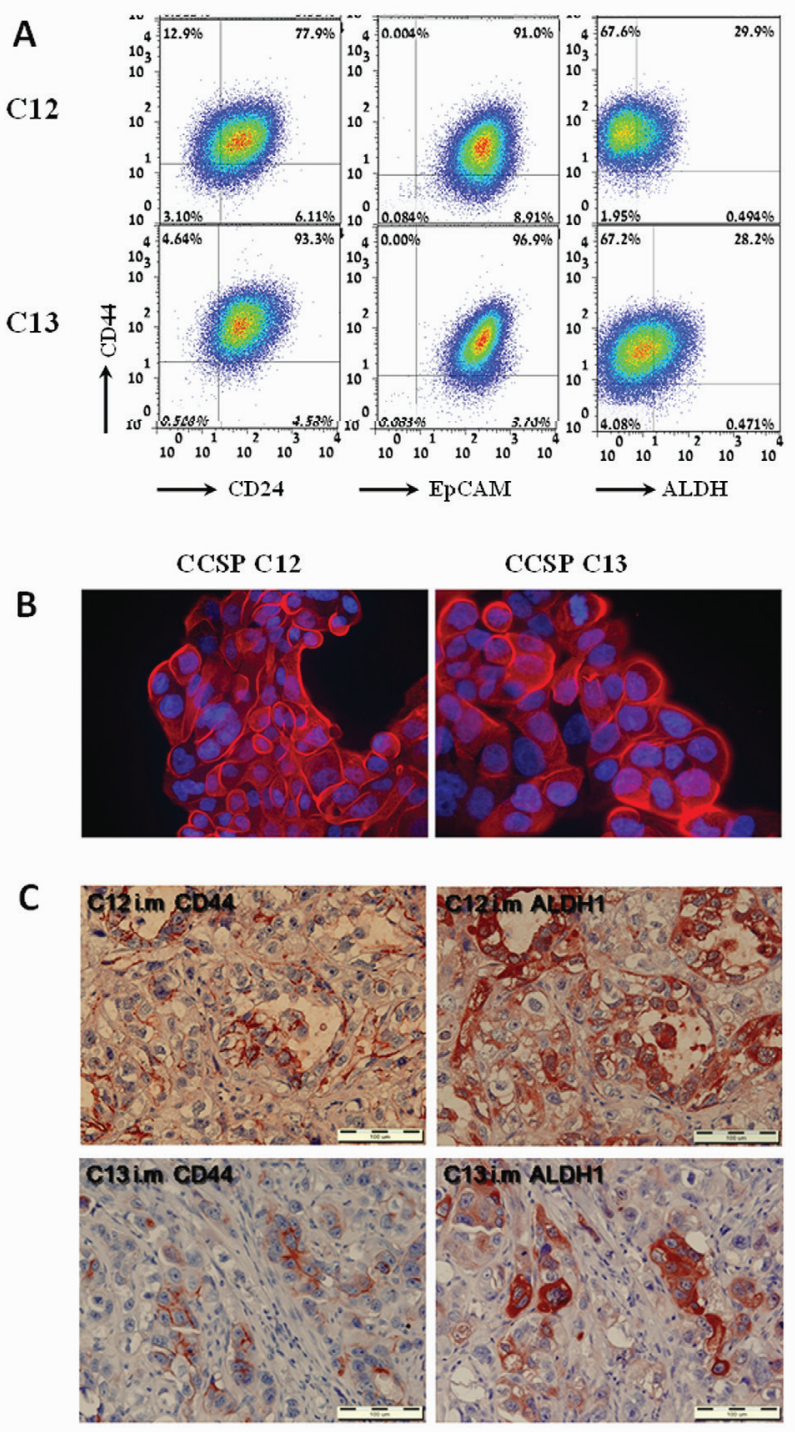

FIG. 2: Expression of stem cell markers in ovarian-derived cancer cell subpopulations (CCSPs) in vitro and in vivo. (A) A surface phenotype of CD44+CD24+EpCAM+for CCSP C12 and C13 was determined by flow cytometry analysis using PE-conjugated anti-CD24, APC-conjugated anti-CD44 and Alexa 488-conjugated anti-EpCAM antibodies. CD44+ALDH1+ cell populations were identified using APC-conjugated anti-CD44 and ALDH1 enzymatic activity. ALDH1 activity was measured with and without ALDH1 inhibitor $\mathrm{N}, \mathrm{N}$-diethylaminobenzaldehyde (DEAB). (B) CD44 expression on CCSP C12 and $\mathrm{C} 13$ cell surface was also confirmed in vitro by immunofluorescence analysis. Bar, $100 \mu \mathrm{m}$. (C) Immunohistochemistry analysis using anti-CD44 and anti-ALDH1 antibodies revealed parallel stained cell clusters in serial section of $\mathrm{C} 12$ and $\mathrm{C} 13$ tumors generated intramuscularly in SCID/Beige mice. Bars, $200 \mu \mathrm{m}$. 
A



B

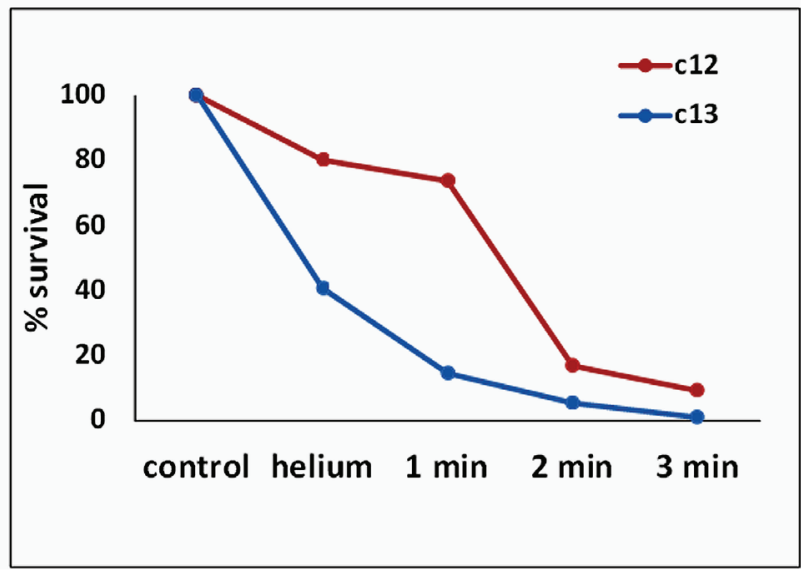

FIG. 3: Effect of CAP treatment on ovarian-derived heterogeneous cancer stem cell (CSC) subpopulations. Ovarian-derived CSC C12 and C13 were treated with CAP for 1,2 , and 3 minutes at days 5,6 , and 8 following seeding as indicated. For control, untreated cells and helium-treated cells were used. (A) Cells were harvested on day 11 (from seeding) and counted. (B) Survival percentage was calculated in comparison with control untreated cells.

\section{Discussion and Concluding Remarks}

Over the last several years, convincing evidence of the efficacy of CAP in cancer treatment has accumulated. ${ }^{4,5}$ Various aspects of CAP-based cancer therapy have been studied worldwide, including the role of reactive species (reactive oxygen and nitrogen), cell cycle modification, in vivo application for solid tumors, CAP interaction with cancer cells in conjunction with nanoparticles, and most recently the first clinical application. ${ }^{4,5,20}$ To this end, the two best known effects of plasma, which are plasma-induced apoptosis and slower cell migration, have important implications in cancer treatment.

Volume 5, Issue 1, 2015 
CAP treatment may help to restrict the spread of cancer and decrease metastatic processes. In this study, we explored the role of CAP in treating CSC, which is another exciting application of this new therapy.

Intratumoral heterogeneity challenges existing paradigms for anticancer therapy. If such heterogeneity also includes self-renewing cells, which sustain the tumor mass, feed into progressive tumorigenic differentiation, and account for tumor recurrence, then attempts to eradicate a single stable self-renewing subpopulation within any given tumor will prove futile. Therefore, any novel method that leads to the destruction of these CSCs will increase the success of cancer treatments.

The molecular mechanisms and cancer cell responses to the CAP jet are not well understood. Normal cells often produce substantially less reactive oxygen and nitrogen species (RONS) than tumor cells. ${ }^{4,5}$ Thus, increased amounts of RONS from the CAP jet could make a dramatic difference in the response of normal and cancer cells to CAP, thereby crossing this survival threshold for tumor cells and leading to cell death through DNA damage, apoptosis, or cell cycle arrest. One plausible hypothesis is that the effect of CAP on CSCs described in this study is associated with RONS generation leading to oxidative stress. Thus, future studies should involve intracellular measurements of RONS.

The results obtained in this study demonstrate that both types of heterogeneous CSC populations derived from a single tumor are sensitive to the effects of plasma treatment, albeit to varying degrees. One interesting outcome is that $\mathrm{C} 13$, which was the more aggressive CSC population, was more sensitive to CAP treatment than $\mathrm{C} 12$. C13 cells are smaller and have a higher proliferation capacity than $\mathrm{C} 12$ cells, and we postulate that these characteristics might increase their sensitivity to CAP. Taken together, our results demonstrate the sensitivity of heterogeneous populations of CSCs derived from a single ovarian tumor to CAP treatment. Our data also suggest that CAP is an efficient and effective anticancer treatment. Further development of CAP technology for CSC treatment should include in vivo studies.

\section{ACKNOWLEDGMENTS}

This research was supported by grants from the Daniel M. Soref Charitable Trust, the Skirball Foundation, the Richard D. Satell Foundation, the Sohnis and Forman families, and the Israel Science Foundation (grant No.62/10 MT).

\section{REFERENCES}

1. Laroussi M, Kong M, Morfill G, Stolz W, editors. Plasma medicine. Cambridge, UK: Cambridge University Press; 2012.

2. Fridman A, Friedman G. Plasma medicine. New York: Wiley; 2013.

3. Keidar M, Beilis II. 2013 Plasma engineering: applications from aerospace to bio and nanotechnology. Waltham, MA: Academic Press; 2013.

4. Keidar M. Plasma for cancer treatment. Plasma Sources Sci Technol. 2015;24:033001. 
5. Ratovitski EA, X. Cheng X, Yan D, J. H. Sherman JH, J. Canady J, B. Trink B, Keidar M. Anti-cancer therapies of 21st century: Novel approach to treat human cancers using cold atmospheric plasma. Plasma Process Polym. 2014;11:1128-37.

6. Vandamme M, Robert E, Pesnel S, Barbosa E, Dozias S, Sobilo J, Lerondel S, Le Pape A, Pouvesle JM. Antitumor effect of plasma treatment on U87 glioma xenografts: preliminary results. Plasma Process Polym. 2010;7:264-73.

7. Vandamme M, Robert E, Lerondel S, Sarron V, Ries D, Dozias S, Sobilo J, Gosset D, Kieda C, Legrain B, Pouvesle JM, Pape AL. ROS implication in a new antitumor strategy based on non-thermal plasma. Int J Cancer. 2012;130:2185-94.

8. Keidar M, Walk R, Shashurin A, Srinivasan P, Sandler A, Dasgupta S, Ravi R, GuerreroPreston R, Trink B. Cold plasma selectivity and the possibility of a paradigm shift in cancer therapy. Br J Cancer. 2011;105:1295-301.

9. Abelson S, Shamai Y, Berger L, Shouval R, Skorecki K, Tzukerman M. Intratumoral heterogeneity in the self-renewal and tumorigenic differentiation of ovarian cancer. Stem Cells. 2012;30:415-24.

10. Abelson S, Shamai Y, Berger L, Skorecki K, Tzukerman M. Niche-dependent gene expression profile of intratumoral heterogeneous ovarian cancer stem cell populations. PloS One. 2013;8:e83651.

11. Kreso A, O'Brien CA, van Galen P, Gan OI, Notta F, Brown AM, Ng K, Ma J, Wienholds E, Dunant C, Pollett A, Gallinger S, McPherson J, Mullighan CG, Shibata D, Dick JE. Variable clonal repopulation dynamics influence chemotherapy response in colorectal cancer. Science. 2013;339:543-8.

12. O'Connor JP, Rose CJ, Waterton JC, Carano RA, Parker GJ, Jackson A. Imaging intratumor heterogeneity: role in therapy response, resistance, and clinical outcome. Clin Cancer Res. 2015;21:249-57.

13. Marusyk A, Polyak K. Tumor heterogeneity: causes and consequences. Biochim Biophys Acta. 2010;1805:105-17.

14. Polyak K, Haviv I, Campbell IG. Co-evolution of tumor cells and their microenvironment. Trends Genet. 2009;25:30-8.

15. Shackleton M, Quintana E, Fearon ER, Morrison SJ. Heterogeneity in cancer: cancer stem cells versus clonal evolution. Cell. 2009;138:822-9.

16. Yap TA, Gerlinger M, Futreal PA, Pusztai L, Swanton C. Intratumor heterogeneity: seeing the wood for the trees. Sci Transl Med. 2012;4:127ps10.

17. Reya T, Morrison SJ, Clarke MF, Weissman IL. Stem cells, cancer, and cancer stem cells. Nature. 2001;414:105-11.

18. Qiu H, Fang X, Luo Q, Ouyang G. Cancer stem cells: a potential target for cancer therapy. Cell Mol Life Sci. 2015;72:3411-24.

19. Walk R M, Snyder J A, Srinivasan P, Kirsch J, Diaz S O, Blanco F C, Shashurin A, Keidar M, Sandler AD. Cold atmospheric plasma for the ablative treatment of neuroblastoma. J Pediatr Surg. 2013;48:67-73.

20. Keidar M, Shashurin A, Volotskova O, Stepp MA, Srinivasan P, Sandler A, Trink B. Cold atmospheric plasma in cancer therapy. Phys Plasmas. 2013; 20:057101.

21. Ikeda J. Effect of nonequilibrium atmospheric pressure plasma on cancer-initiating cells. Plasma Medicine. 2014;4:49-56.

22. Katz E, Skorecki K, Tzukerman M. Niche-dependent tumorigenic capacity of malignant ovarian ascites-derived cancer cell subpopulations. Clin Cancer Res. 2009;15:70-80.

Volume 5, Issue 1, 2015 
23. Shashurin A, Keidar M, Bronnikov S, Jurjus RA, Stepp MA. Appl Phys Lett. 2008;92:181501

24. Czernobilsky B, Silverman BB, Enterline HT. Clear-cell carcinoma of the ovary. A clinicopathologic analysis of pure and mixed forms and comparison with endometrioid carcinoma. Cancer. 1970;25: 762-72.

25. Montag AG, Jenison EL, Griffiths CT, Welch WR, Lavin PT, Knapp RC. Ovarian clear cell carcinoma. A clinicopathologic analysis of 44 cases. Int J Gynecol Pathol. 1989;8:85-96.

26. Tan DS, Kaye S. Ovarian clear cell adenocarcinoma: a continuing enigma. J Clin Pathol. 2007;60:355-60.

27. Kobel M, Kalloger SE, Carrick J, Huntsman D, Asad H, Oliva E, Ewanowich CA, Soslow RA, Gilks CB. A limited panel of immunomarkers can reliably distinguish between clear cell and high-grade serous carcinoma of the ovary. Am J Surg Pathol. 2009;33:14-21.

28. DeLair D, Oliva E, Kobel M, Macias A, Gilks CB, Soslow RA. Morphologic spectrum of immunohistochemically characterized clear cell carcinoma of the ovary: a study of 155 cases. Am J Surg Pathol. 2011;35:36-44. 